\title{
MicroRNA-296-5p inhibits cell proliferation by targeting HMGA1 in colorectal cancer
}

\author{
GUOHUI YAN ${ }^{1-3^{*}}$, SHUIDI YAN $^{4 *}$, JIAJIA WANG $^{4}$, SHEN LEI $^{2}$, \\ WEIMIN TIAN $^{5}$, XIN YUE $^{6}$ and YANG ZHANG ${ }^{1,2,4}$

\begin{abstract}
${ }^{1}$ The Medical Department of the Xiamen University, Xiamen, Fujian 361000; ${ }^{2}$ The Medical Department of the Fujian Medical University, Fuzhou, Fujian 350000; ${ }^{3}$ Department of Ultrasound; ${ }^{4}$ Center of Clinical Laboratory, Departments of ${ }^{5}$ Paediatrics and ${ }^{6}$ Imaging, Zhongshan Hospital, School of Medicine,

Xiamen University, Xiamen, Fujian 361004, P.R. China
\end{abstract}

Received February 20, 2019; Accepted November 8, 2019

DOI: $10.3892 /$ etm.2021.10225

\begin{abstract}
An increasing body of evidence indicates the involvement of microRNAs (miRNAs/miRs) in the initiation and progression of colorectal cancer (CRC). miR-296-5p was recently identified as a tumor suppressor in a variety of human cancer types; however, its function in CRC remains largely unknown. The present study demonstrated that the expression of miR-296-5p was significantly downregulated in CRC tissues and cell lines. The overexpression of miR-296-5p markedly inhibited proliferation, and induced cell cycle arrest and apoptosis in CRC cells. Bioinformatics analysis suggested that high mobility group AT-hook 1 (HMGA1) may be a target of miR-296-5p in CRC cells. Further experiments showed that miR-296-5p bound the 3'-untranslated region of HMGA1 and decreased its expression in CRC cells. HMGA1 was overexpressed in CRC tissues and was inversely correlated with the expression of miR-296-5p. The restoration of HMGA1 significantly reversed the inhibitory effect of miR-296-5p on the proliferation of CRC cells. Overall, the findings of the present study indicate that miR-296-5p suppressed the progression of CRC, at least partially via targeting HMGA1. Thus, miR-296-5p is a potential target for novel therapies in CRC.
\end{abstract}

Correspondence to: Dr Yang Zhang, Center of Clinical Laboratory, Zhongshan Hospital, School of Medicine, Xiamen University, 201-209 Hu Bin South Road, Xiamen, Fujian 361004, P.R. China

E-mail: buzuo823677@163.com

${ }^{*}$ Contributed equally

Key words: microRNA-296-5p, colorectal cancer, high mobility group AT-hook 1 , cell cycle

\section{Introduction}

Colorectal cancer (CRC) is considered to be one of the most common causes of cancer-associated mortality worldwide $(1,2)$. Although therapeutic strategies, including surgical resection, chemotherapy and radiotherapy, have greatly improved the outcome of patients with CRC, the treatment of patients with $\mathrm{CRC}$ requires further improvement (3). Therefore, more efforts should be made in investigating the molecular mechanisms involved in the development of $\mathrm{CRC}$, which might provide novel targets and therapeutic methods for the treatment of CRC.

MicroRNAs (miRNAs/miRs) are defined as a category of endogenous single-stranded, non-coding RNAs that are 21-23 nucleotides in length (4-6). miRNAs act as key negative regulators of gene expression, via binding to the 3'-untranslated regions (3'-UTRs) of target mRNAs, which consequently inhibit the translation or induce the degradation of mRNAs (7). Increasing evidence has indicated that miRNAs play essential roles in diverse cellular processes, including cell proliferation, differentiation and apoptosis (8). The vital function of miRNAs in the progression of human cancer was also demonstrated by recent studies (9-12). miRNAs modulate the progression of cancer by acting as oncogenes or tumor suppressors $(9,12-16)$. Recently, miR-26b was found to improve the sensitivity of CRC cells to 5-flurouracil and inhibited the growth of CRC cells (17). Wang et al (18) reported that miR-410 promoted the malignancy of CRC and could be used as a potential biomarker in the progression of CRC. Inhibiting the expression of miR-30d promoted the cell proliferation and tumor growth of CRC by targeting G protein subunit $\alpha 13$ (19). miR-296-5p was recently reported as a tumor suppressor in non-small cell lung cancer by directly targeting polo-like kinase 1 (PLK1) (20). Similarly, miR-296-5p suppressed the proliferation of prostate cancer cells, implicating the potential application of miR-296-5p in the prognosis of prostate cancer (21). Although previous reports have demonstrated the tumor suppressive role of miR-296-5p in cancer, the function and molecular mechanism of miR-296-5p in CRC remains largely unknown.

The present study aimed to investigate the possible role of miR-296-5p in the progression of CRC and characterize the potential underlying molecular mechanism. 


\section{Materials and methods}

Tissue samples and cell lines. A cohort of 40 CRC tissues and corresponding adjacent normal tissues $(5 \mathrm{~cm}$ from the tumor margin and histologically confirmed) were collected from CRC patients (female: male $=1.86: 1$; age range, 39-72 years; mean age, 61.4 years) at Zhongshan Hospital (Xiamen, China), via surgical resection between May 2012 and September 2014. None of these patients received chemotherapy, radiotherapy and immunotherapy prior to the tissue collection. Tissues were stored at $-80^{\circ} \mathrm{C}$ before further experiments. Tumors sample were staged according to the National Comprehensive Cancer Network guidelines (22). Informed consent was obtained from all patients. This study was approved by the Institutional Ethics Committee of Zhongshan Hospital, Xiamen University.

The CRC cell lines (HCT116, HT-29, LOVO) and human normal colorectal epithelial cell (FHC) were purchased from the American Type Culture Collection. The cells were cultured in DMEM (Thermo Fisher Scientific, Inc.), supplemented with 10\% FBS (Gibco; Thermo Fisher Scientific, Inc.), $100 \mathrm{U} / \mathrm{ml}$ penicillin and $100 \mu \mathrm{g} / \mathrm{ml}$ streptomycin, at $37^{\circ} \mathrm{C}$ with $5 \% \mathrm{CO}_{2}$.

Reagents. The antibodies used in this study included: Anti-HMGA1 (cat. no. 7777; Cell Signaling Technology, Inc.), anti-GAPDH (cat. no. G8795; Sigma-Aldrich; Merck KGaA) and HRP-conjugated secondary antibody (cat. no. 7047; Cell Signaling Technology, Inc.).

Cell transfection. The miR-296-5p mimics (5'-AGGGCCCCCCCUCAAUCCUGU), control miRNA (5'-GGUUCGUACGUACACUGUUCA), miR-296-5p antagomir (5'-UCCCGGGGGGGAGUUAGGACA-3') and the negative control miRNA (5'-CGGUACGAUCGCGGC GGGAUAUC-3') were synthesized by Shanghai GenePharma Co.,Ltd. Both the negative control miRNAs were non-targeting sequences. To construct Flag-tagged HMGA1, the full-length of HMGA1 cDNA was obtained by reverse transcription from RNA samples extracted form HCT116 cells and ligated into the c-Flag pcDNA3 vector (cat. no. 20011; Addgene, Inc.) at the EcoRI and XhoI sites. A total of $20 \mathrm{nM}$ miRNAs and/or $0.5 \mu \mathrm{g}$ of Flag-HMGA1 or Flag-vector were transfected into HCT116 and HT-29 cells, seeded at the density of 10,000 cells/well using Lipofectamine $^{\circledR} 2000$ (Invitrogen; Thermo Fisher Scientific, Inc.) according to the manufacturer's instructions. Following transfection for $48 \mathrm{~h}$, the cells were harvested for the subsequent experiments.

Reverse transcription-quantitative (RT-q)PCR. The extraction of RNA from CRC tissues and cell lines was performed using the TRIzol ${ }^{\circledR}$ reagent (Invitrogen; Thermo Fisher Scientific, Inc.). RNA was reverse transcribed into cDNA using the miScript RT kit (Takara Biotechnology Co., Ltd.), according to the manufacturer's protocols using the temperature protocol of $25^{\circ} \mathrm{C}$ for $5 \mathrm{~min}, 46^{\circ} \mathrm{C}$ for $20 \mathrm{~min}$ and $95^{\circ} \mathrm{C}$ for $1 \mathrm{~min}$. qPCR was performed to determine the expression of miR-296-5p or HMGA1, using SYBR Green mix (Bio-Rad Laboratories, Inc.) on the Applied Biosystems 7500 Sequence Detection System (Applied Biosystems; Thermo Fisher Scientific, Inc). The primers used in this study were as follows: miR-296-5p forward, 5'-GTATCCAGTGCAGGGTCCGA-3'; miR-296-5p reverse, 5'-CGACGAGGGCCCCCCCT-3'; U6 RNA forward, 5'-CGAGCACAGAATCGCTTCA-3'; U6 RNA reverse, 5'-CTCGCTTCGGCAGCACATAT-3'; HMGA1 forward, 5'-CAACTCCGGGGAGGAAACCA-3'; HMGA1 reverse, 5'-AGGACTCCTGGGAGATGC-3'; GAPDH forward, 5'-GCCTTCTCCATGGTGGTGAA-3'; and GAPDH reverse, 5'-GGTCGGTGTGAACGGATTTG-3'. The PCR conditions were as follows: $95^{\circ} \mathrm{C}$ for $10 \mathrm{~min}$, followed by 40 cycles of $95^{\circ} \mathrm{C}$ for $10 \mathrm{sec}$ and $58^{\circ} \mathrm{C}$ for $60 \mathrm{sec}$. The relative expression of miR-296-5p and HMGA1 was calculated using the 2- $-\Delta \Delta \mathrm{Cq}$ method (23) and was normalized to the expression of U6 RNA or GAPDH, respectively.

Targets prediction. The potential targets of miR-296-5p were predicted using the miRDB (http://mirdb.org/) (24) and TargetScan databases (release 7.2; http://www.targetscan. org/vert_72/).

Western blot analysis. Protein was extracted by lysing the CRC cells using NP-40 buffer (Beyotime Institute of Biotechnology) containing protease inhibitors (Roche Diagnostics). Protein concentration was quantified using the bicinchoninic acid method (Beyotime Institute of Biotechnology). A total of $20 \mu \mathrm{g}$ protein was separated by SDS-PAGE on a $15 \%$ gel, and transferred onto a PVDF membrane (EMD Millipore). After blocking with 5\% non-fat milk at room temperature (RT) for $1 \mathrm{~h}$, the membrane was incubated with anti-HMGA1 $(1: 1,000)$, anti-GAPDH $(1: 3,000)$ or anti-Flag $(1: 3,000$; cat. no. F7425; Sigma-Aldrich; Merck KGaA) primary antibodies overnight at $4^{\circ} \mathrm{C}$. After washing twice with PBS, the membrane was incubated with horseradish peroxidase (HRP)-conjugated secondary antibody $(1: 10,000)$ for $1 \mathrm{~h}$ at RT. The blot signals were visualized with enhanced chemiluminescence chromogenic substrate (EMD Millipore), according to the manufacturer's instructions. The expression of GAPDH was determined as the loading control. The protein bands were analyzed with Image J analysis software (v.1.52n; National Institutes of Health). Western blot analysis was performed three times independently.

Ultrasound-mediated microbubble destruction. The doxorubicin (Dox)-liposome-microbubble complex (DLMC) was prepared as previously described (19). To induce the uptake of Dox, CRC cells were seeded in a 24-well plate and incubated overnight at $37^{\circ} \mathrm{C}$. Cells were subsequently incubated with DLMC containing $10 \mu \mathrm{g} / \mathrm{ml}$ Dox, and ultrasound (US) radiation was applied for $15 \mathrm{sec}$ by moving a $20 \mathrm{~mm}$ US probe E1609 (Valpey Fisher, Inc.) over the cell culture plate with the following parameters: $1 \mathrm{MHz}, 20 \%$ duty cycle, US intensity of $1.65 \mathrm{~W} / \mathrm{cm}^{2}$ and US peak intensity of $0.35 \mathrm{MPa}$, as described previously (25). The cells were then rinsed with serum-free DMEM to remove the uninternalized Dox. Cells with DLMC containing $10 \mu \mathrm{g} / \mathrm{ml}$ of Dox without US treatment were investigated as the mock. Cells were then cultured with fresh medium overnight at $37^{\circ} \mathrm{C}$ and subjected to subsequent experiments.

Cell Counting Kit-8 (CCK-8) assay. Both HCT116 and HT-29 cells were plated in the 96-well plate at the density of 1,000 cells per well. Following culture for $24 \mathrm{~h}$, the cells were transfected with miR-296-5p mimics or control miRNA using 
Lipofectamine ${ }^{\circledR} 2000$ (Invitrogen; Thermo Fisher Scientific, Inc.). After $24 \mathrm{~h}, 10 \mu \mathrm{l}$ CCK-8 solution (Dojindo Molecular Technologies, Inc.) was added into the medium and incubated at $37^{\circ} \mathrm{C}$ for an additional $3 \mathrm{~h}$, according to the manufacturer's protocol. The absorbance of each well at $450 \mathrm{~nm}$ was measured using a microplate reader (Bio-Rad Laboratories, Inc.). The experiments were performed in triplicate.

Luciferase reporter assay. The 3'-UTR of HMGA1 containing the putative binding sites of miR-296-5p was amplified from human genomic DNA and constructed into the pGL3 luciferase reporter vector (Promega Corporation). A total of $0.5 \mu \mathrm{g}$ luciferase vector encoding the wild-type or mutant 3'-UTR, and miR-296 mimics or control miRNA were co-transfected into the CRC cells (density, 10,000 cells/well) with Lipofectamine ${ }^{\circledR}$ 2000 (Invitrogen; Thermo Fisher Scientific, Inc.). After transfection for $48 \mathrm{~h}$, the cells were harvested and the luciferase activity was determined using the Dual-Luciferase Reporter Assay System (Promega Corporation). The activity of Renilla luciferase was detected for normalization.

Cell apoptosis analysis. Both HCT116 and HT-20 cells were seeded on a 6-well plate and transfected with miR-296-5p mimics or control miRNA. After transfection for $48 \mathrm{~h}$, the percentage of cell apoptosis was evaluated using the Annexin-V FITC Apoptosis Detection kit (Invitrogen; Thermo Fisher Scientific, Inc.), according to the manufacturer's instructions. Cell apoptosis was determined using a flow cytometer (BD Biosciences). Data analysis was performed using ModFit software (v.3.3; BD Biosciences).

Cell cycle analysis. Cells were cultured with serum-free medium for $24 \mathrm{~h}$ and then transfected with miR-296-5p mimics or control miRNA for $48 \mathrm{~h}$ with medium containing $10 \%$ FBS. The transfected cells were harvested and washed twice with pre-cooled PBS and fixed with $70 \%$ ethanol overnight at $4^{\circ} \mathrm{C}$. After washing with PBS, the cells were stained with $100 \mu \mathrm{g} / \mathrm{ml}$ propidium iodide and $50 \mu \mathrm{g} / \mathrm{ml} \mathrm{RNase}$ for $30 \mathrm{~min}$ at RT in the dark. The cell cycle was detected with a flow cytometer (BD Biosciences). The profile was analyzed using ModFit software (v.3.3; BD Biosciences).

Statistical analysis. Data are presented as the mean \pm standard deviation and were analyzed with the GraphPad Prism software (v.5.0; GraphPad Software, Inc.). The differences between two groups were analyzed using the Student's t-test; the differences between normal colon and colon cancer tissues were analyzed by paired t-test. The comparisons between three or more groups were assessed using the one-way analysis of variance, followed by Tukey's test. The correlation between the expression of miR-296-5p and HMGA1 was determined by the Spearman test. The association between the level of miR-296-5p and the clinical features of patients with CRC was analyzed using the $\chi^{2}$ test. $\mathrm{P}<0.05$ was considered to indicate a statistically significance difference.

\section{Results}

Expression of miR-296-5p is downregulated in CRC. To evaluate the potential involvement of miR-296-5p in CRC, the
Table I. Association between the expression of miR-296-5p and the clinicopathological characteristics of patients with colorectal carcinoma.

\begin{tabular}{lcccc}
$\begin{array}{l}\text { Clinical } \\
\text { characteristics }\end{array}$ & Cases, $\mathrm{n}$ & Low, $\mathrm{n}$ & High, $\mathrm{n}$ & P-value \\
\hline $\begin{array}{l}\text { Age, years } \\
\leq 60\end{array}$ & 14 & 10 & 4 & 0.345 \\
$>60$ & 26 & 18 & 8 & \\
Sex & & & & 0.176 \\
Male & 19 & 15 & 4 & \\
Female & 21 & 13 & 8 & \\
Tumor size, cm & & & & 0.004 \\
$\geq 5$ & 24 & 19 & 5 & \\
$<5$ & 16 & 9 & 7 & \\
TNM stage & & & & $<0.001$ \\
I + II & 15 & 7 & 8 & \\
III + IV & 25 & 21 & 4 & \\
Metastasis & & & & $<0.001$ \\
Yes & 27 & 22 & 5 & \\
No & 13 & 6 & 7 &
\end{tabular}

TNM, tumor-node-metastasis.

expression of miR-296-5p in CRC tissues and matched adjacent normal tissues was detected by RT-qPCR. The results showed that the expression of miR-296-5p was significantly decreased in CRC tissues compared with adjacent non-tumor tissues (Fig. 1A). Consistently, the downregulation of miR-296-5p was also observed in CRC cell lines, including HCT116, HT-29 and LOVO, compared with the expression of miR-296-5p in normal FHC cells (Fig. 1B). To further characterize the association between the expression of miR-296-5p and the prognosis of patients with CRC, all 40 patients enrolled in the present study were divided into a miR-296-5p high and low group, according to the mean value (3.85) of miR-296-5p expression. The results showed that low expression of miR-296-5p was significantly associated with a higher Tumor-Node-Metastasis stage, lymph node metastasis and tumor size, suggesting the potential clinical significance of miR-296-5p in CRC (Table I). The decreased expression of miR-296-5p in CRC cells compared with non-cancerous colon tissues and cell lines suggested the potential role of miR-296-5p in CRC.

Overexpression of miR-296-5p inhibits proliferation and induces apoptosis in CRC cells. In order to explore the regulatory role of miR-296-5p in CRC progression, HCT116 and HT-29 cells were transfected with miR-296-5p mimics or control miRNA. As presented in Fig. 2A, compared with the control cells, the expression of miR-296-5p was significantly increased in both HCT116 and HT-29 cells following the transfection with miR-296-5p mimics. The CCK-8 assay was performed to determine the effect of miR-296-5p on the proliferation of CRC cells. The data showed that the overexpression of miR-296-5p significantly inhibited the proliferation of both HCT116 and HT-29 
A

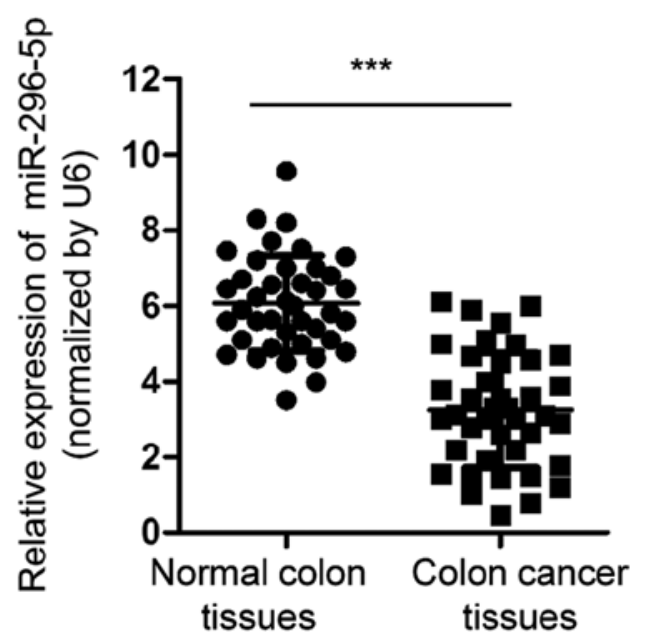

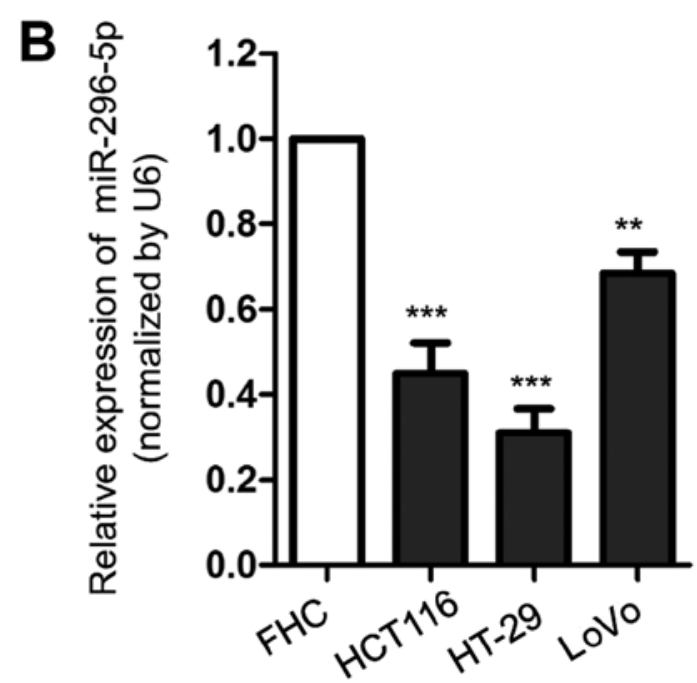

Figure 1. miR-296-5p is downregulated in CRC. (A) The expression of miR-296-5p in paired CRC tissues and corresponding normal tissues was detected by reverse transcription-quantitative PCR. ${ }^{* * *} \mathrm{P}<0.001$. (B) The expression level of miR-296-5p was significantly decreased in CRC cell lines compared with normal FHC cells. ${ }^{* *} \mathrm{P}<0.01,{ }^{* * *} \mathrm{P}<0.001$ vs. FHC. miR, microRNA; CRC, colorectal cancer.

cells (Fig. 2B and C). Additionally, the flow cytometry indicated that the overexpression of miR-296-5p led to G1-phase cell cycle arrest in HCT116 cells (Fig. 2D). In accordance, the apoptosis of CRC cells was also significantly upregulated following the overexpression of miR-296-5p (Fig. 2E).

To further validate the suppressive function of miR-296-5p in CRC, HCT116 and HT-29 cells were treated with Dox via US-mediated microbubble destruction. The results showed that high expression of miR-296-5p and treatment with Dox synergistically inhibited the proliferation of CRC cells (Fig. 2F). These findings suggested the potential tumor suppressive function of miR-296-5p in modulating the growth of CRC cells.

HMGA1 is a target of miR-296-5p in CRC cells. To further investigate the functional mechanism of miR-296-5p in CRC, the potential targets of miR-296-5p were predicted using the miRDB and TargetScan databases. Following the search, HMGA1 was identified as a possible target of miR-296-5p. The putative complementary sequence of miR-296-5p at the 3'-UTR of HMGA1 is presented in Fig. 3A. To further confirm this, the luciferase reporter assay was performed by co-transfecting luciferase vectors harboring the wild-type or mutant 3'-UTR of HMGA1, and miR-296-5p mimics or control miRNA, into both HCT116 and HT-29 cells. The overexpression of miR-296-5p significantly decreased the luciferase activity of the wild-type, but not the mutant, 3'-UTR of HMGA1 (Fig. 3B and C). To determine whether the binding of miR-296-5p with the 3'-UTR of HMGA1 affects the mRNA stability of HMGA1, RT-qPCR was performed following the transfection of HCT116 and HT-29 cells with miR-296-5p mimics or control miRNA. The results showed that the overexpression of miR-296-5p significantly decreased the mRNA levels of HMGA1 in CRC cells (Fig. 3D). Consistently, the protein abundance of HMGA1 was also decreased in both HCT116 and HT-29 cells overexpressing miR-296-5p (Fig. 3E). To further validate the suppressive effect of miR-296-5p on the expression of HMGA1, miR-296-5p was downregulated by transfecting $\mathrm{CRC}$ cells with miR-296-5p antagomir. The transfection efficiency of miR-29605p was validated via RT-qPCR, with the GFP as the transfection control (Fig. 3F). In accordance, the downregulation of miR-296-5p resulted in significantly increased mRNA and protein levels of HMGA1 (Fig. 3G and H). Overall, these results demonstrated that miR-296-5p targeted HMGA1 and negatively modulated the expression levels of HMGA1 in CRC cells.

Overexpression of HMGAl attenuates the suppressive role of miR-296-5p on the proliferation of CRC cells. The expression of HMGA1 in CRC tissues and matched adjacent normal tissues was detected via RT-qPCR. The data showed that the mRNA level of HMGA1 was significantly overexpressed in CRC tissues compared with the adjacent normal tissues (Fig. 4A). As HMGA1 was identified as a target of miR-296-5p, the correlation between the expression of miR-296-5p and HMGA1 in CRC tissues was determined by Spearman analysis. As presented in Fig. 4B, a significantly negative correlation was observed between the level of miR-296-5p and HMGA1 in CRCC tissues.

To further confirm the function of HMGA1 in the tumor suppressive role of miR-296-5, the expression of HMGA1 was restored through transfecting Flag-tagged HMGA1 into both HCT116 and HT-29 cells (Fig. 4C). The CCK-8 assay showed that the transfection of HMGA1 significantly reversed the inhibitory effect of miR-296-5p on the proliferation of both HCT116 and HT-29 cells (Fig. 4D and E). Moreover, the overexpression of HMGA1 eliminated the miR-296-5p-induced apoptosis of CRC cells (Fig. 4F). These results indicated the essential role of HMGA1 in mediating the suppressive function of miR-296-5p in CRC.

\section{Discussion}

Accumulating evidence suggests the critical roles of miRNAs in the initiation and progression of cancer by modulating the expression of target genes (13-15). The aberrant expression 
A

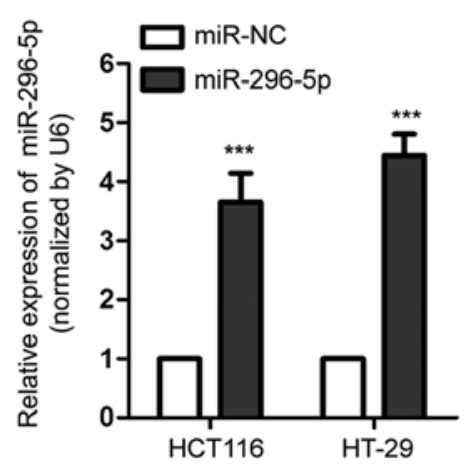

B

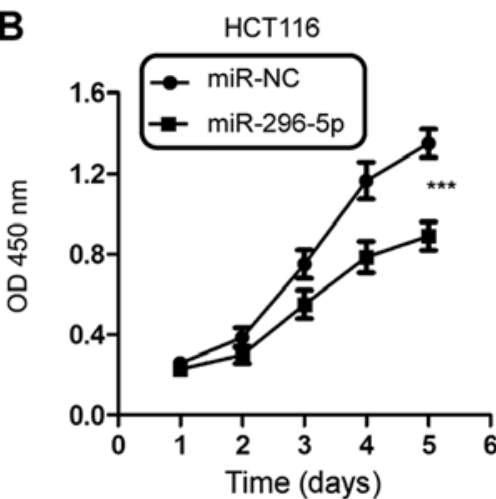

C

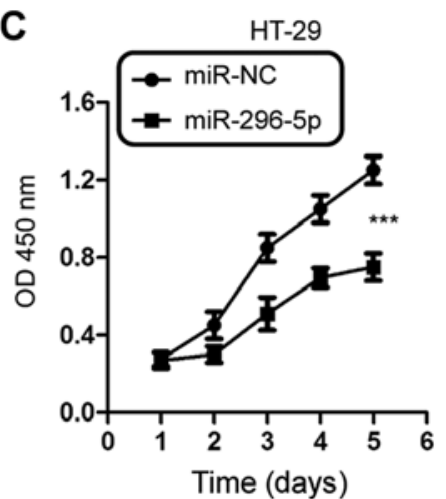

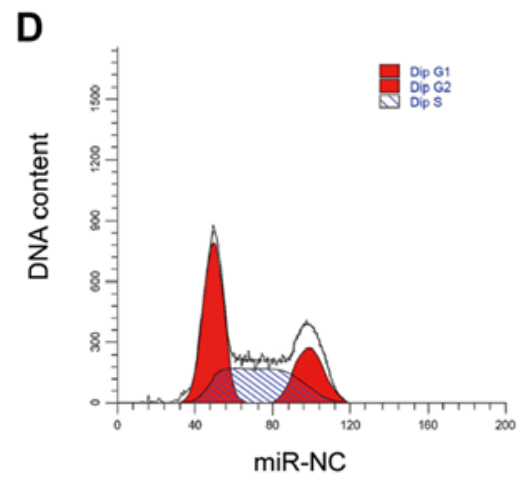
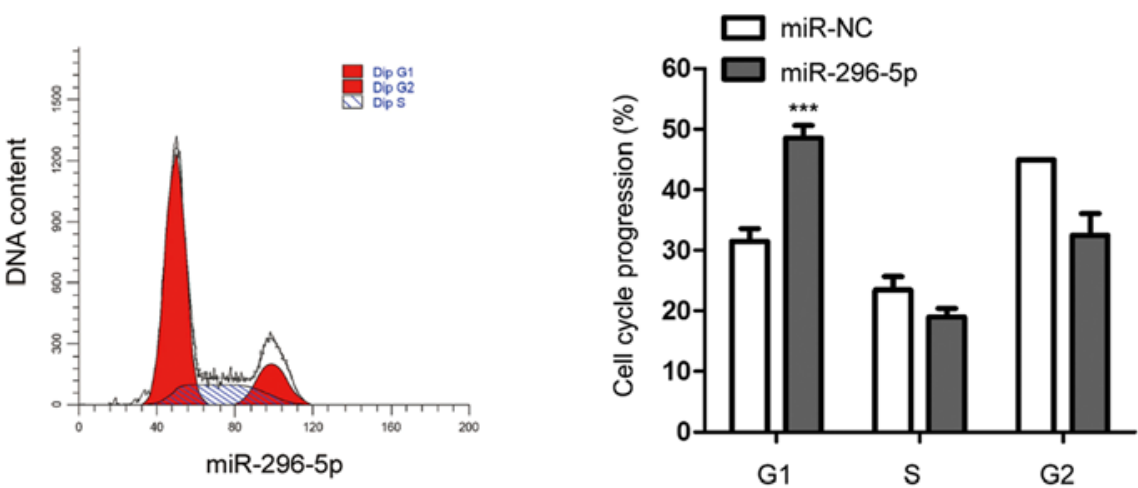

E
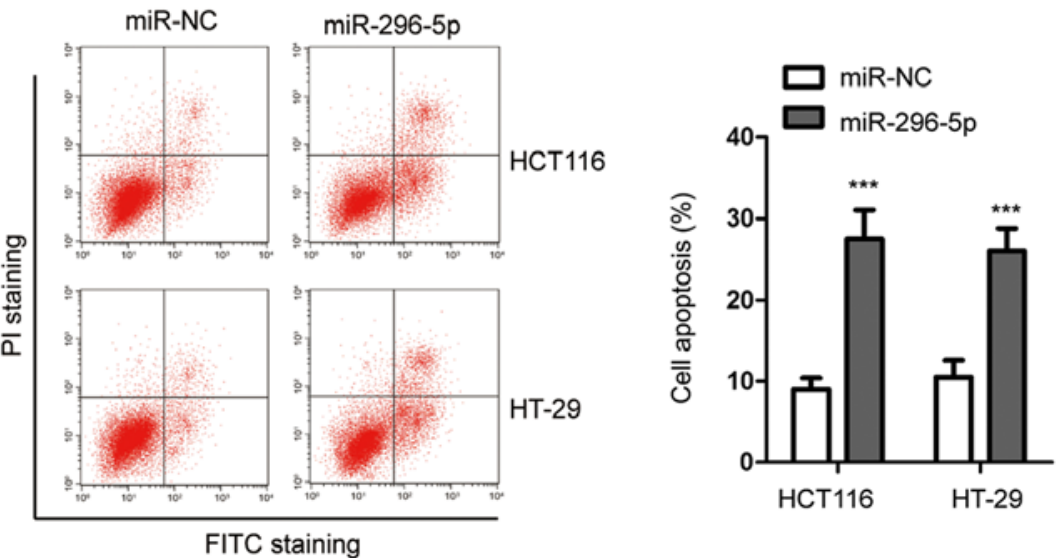

$\mathbf{F}$

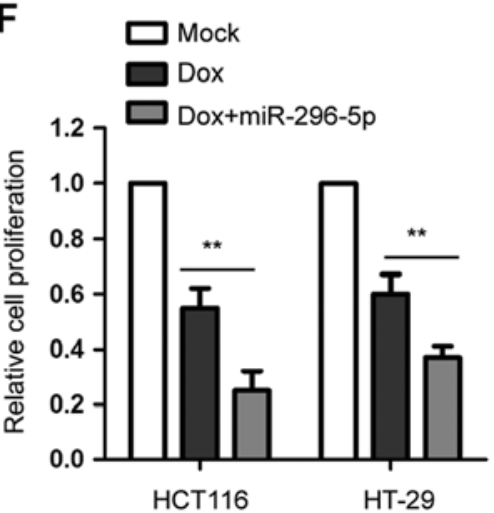

Figure 2. Overexpression of miR-296-5p inhibits the proliferation of CRC cells. (A) Validation of the miR-296-5p expression level after transfection with miR-296-5p mimics or control miRNA for $48 \mathrm{~h}$ in both HCT116 and HT-29 cells. ${ }^{* * *} \mathrm{P}<0.001$ vs. respective miR-NC group. The proliferation of (B) HCT116 and (C) HT-29 cells transfected with miR-296-5p was significantly decreased compared with those transfected with control miRNA. ${ }^{* * *}$ P $<0.001$ vs. respective miR-NC group. (D) The cell cycle progression of colorectal cancer cells was determined by flow cytometry, following the overexpression of miR-296-5p. ${ }^{* * *} \mathrm{P}<0.001$ vs. respective miR-NC group. (E) The apoptosis of HCT116 and HT-29 cells was measured following transfection with miR-296-5p for 48 h. ${ }^{* * *} \mathrm{P}<0.001$ vs. respective miR-NC group. (F) The overexpression of miR-296-5p significantly promoted the inhibitory effect of Dox on the growth of HCT116 and HT-29 cells. ${ }^{* *} \mathrm{P}<0.01$. miR/miRNA, microRNA; CRC, colorectal cancer; Dox, doxorubicin; NC, negative control; PI, propidium iodide.

of miR-296-5p has been found in a number of different types of cancer in humans, including non-small cell lung cancer, prostate cancer and hepatocellular carcinoma $(20,21,26)$. The downregulation of miR-296-5p is associated with a poor prognosis in patients with cancer (27). In the present study, it was found that the expression of miR-296-5p was significantly decreased in CRC tissues and cell lines, suggesting the potential involvement of miR-296-5p in CRC.

The tumor suppressive function of miR-296-5p has been established in several types of cancer. For example, miR-296-5p is prominently downregulated in hepatocellular carcinoma (HCC) and inhibits the epithelial-to-mesenchymal transition of HCC (26). The decreased expression of miR-296-5p is significantly associated with a favorable prognosis in patients with HCC (26). The inhibitory effect of miR-296-5p on the growth of cancer cells has also been observed in non-small cell lung cancer, by targeting PLK1 (20). Additionally, miR-296-5p plays a tumor-suppressive role in prostate cancer by downregulating peptidyl-prolyl cis-trans isomerase NIMA-interacting 1, indicating its potential application in the prognosis of prostate cancer (21). Studies have also demonstrated the promoting effect of miR-296-5p on the development of cancer $(28,29)$. For example, miR-296-5p enhances the invasiveness of glioblastoma by suppressing the 
A

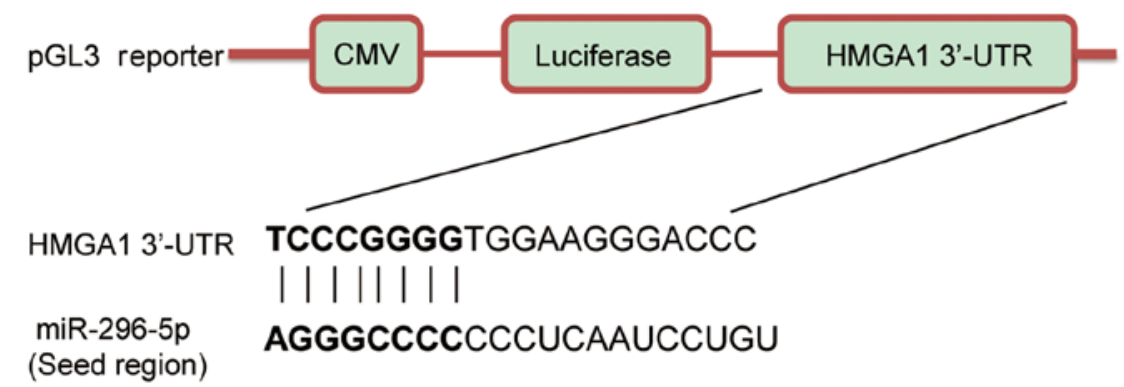

B

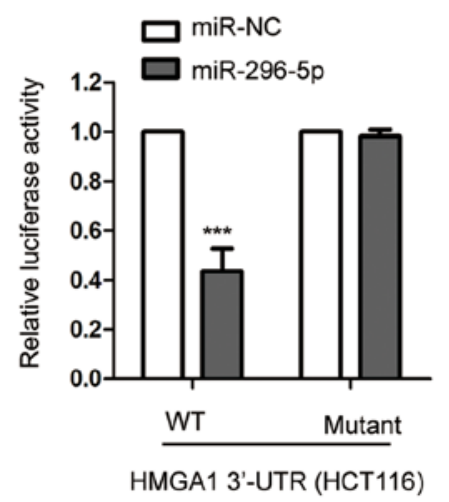

D

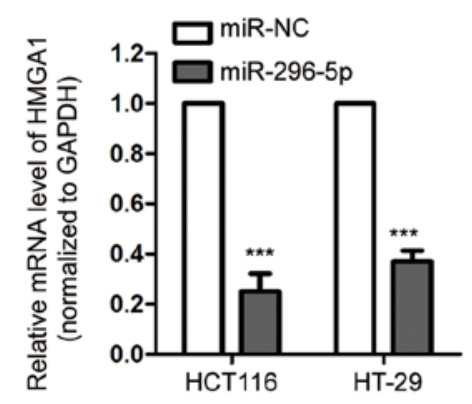

C

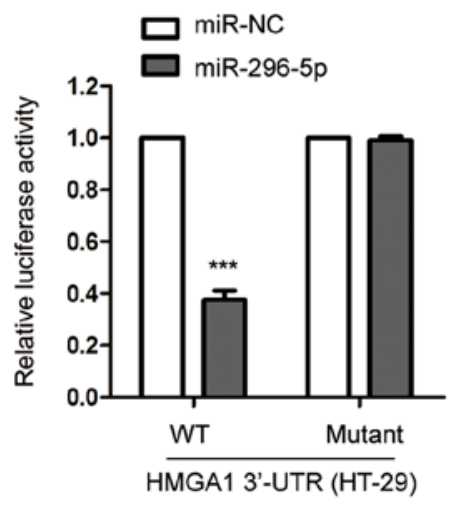

E

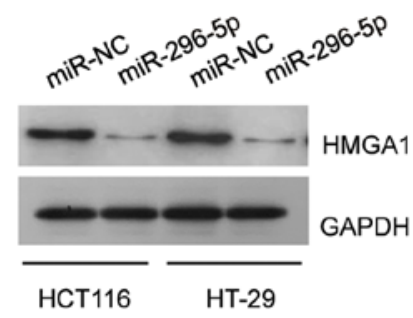

$\mathbf{F}$

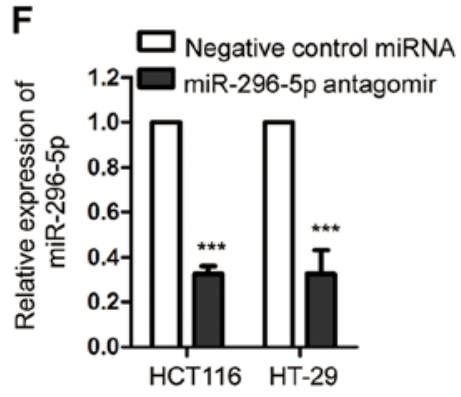

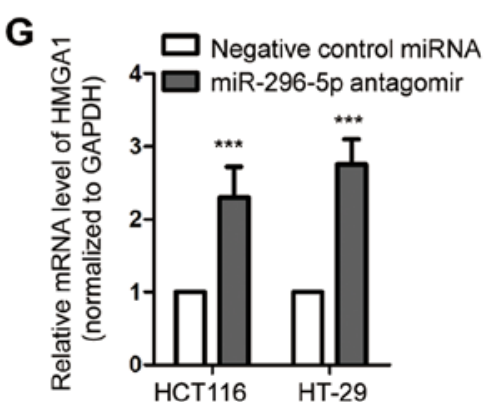

H

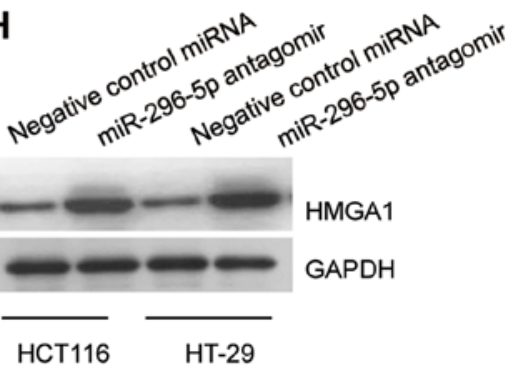

Figure 3. HMGA1 is a target of miR-296-5p in CRC cells. (A) Schematic representation of potential binding sites of miR-296-5p in the 3'-UTR of HMGA1. Dual luciferase activity of CRC cells following transfection with miR-296-5p mimics or control miRNA in (B) HCT116 and (C) HT-29 cells. The (D) mRNA and (E) protein expression of HMGA1 in HCT116 and HT-29 cells, following transfection with miR-296-5p. (F) Transfection with miR-296-5p mimic significantly decreased its expression in CRC cells. The (G) mRNA and (H) protein expression level of HMGA1 in CRC cells, following the overexpression of miR-296-5p by the antagomir. ${ }^{* * * *} \mathrm{P}<0.001$ vs. respective miR-NC group. HMGA1, high mobility group AT-hook 1; miR/miRNA, microRNA; CRC, colorectal cancer; UTR, untranslated region; NC, negative control; WT, wild-type.

expression of nerve growth factor receptor and caspase-8 (29). Similarly, miR-296-5p enhances the cell proliferation of gastric cancer (28). In the present study, miR-296-5p was downregulated in CRC tissues compared with matched normal tissues. The overexpression of miR-296-5p significantly inhibited the proliferation and induced apoptosis of CRC cells. These results indicated the tumor suppressive function of miR-296-5p in the progression of CRC.
As an important transcription factor, HMGA1 is involved in regulating autophagy and cell invasion, which contributes to cancer progression $(30,31)$. Moreover, HMGA1 was found to enhance tumorigenesis by conferring resistance to chemotherapies, including trabectedin, temozolomide, paclitaxel, doxorubicin and antineoplastic drugs (32-35). These findings suggested the potential oncogenic function of HMGA1 in cancer progression. Notably, HMGA1 has been identified as the target 
A

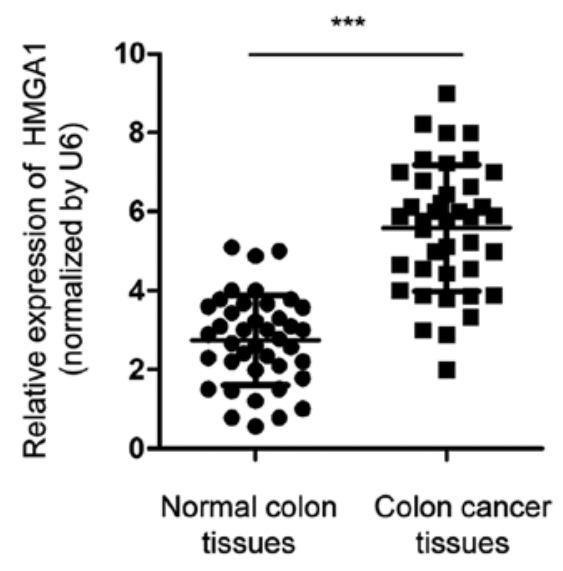

C

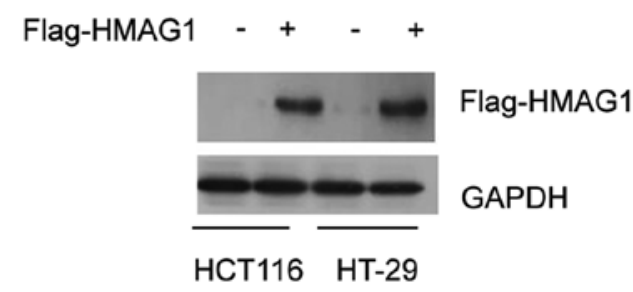

$\mathbf{E}$

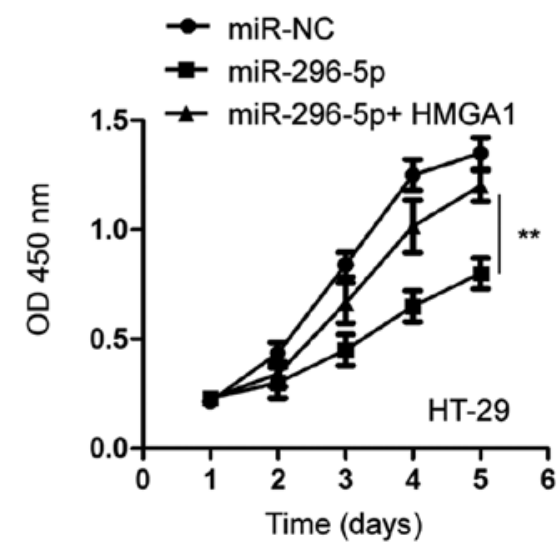

B

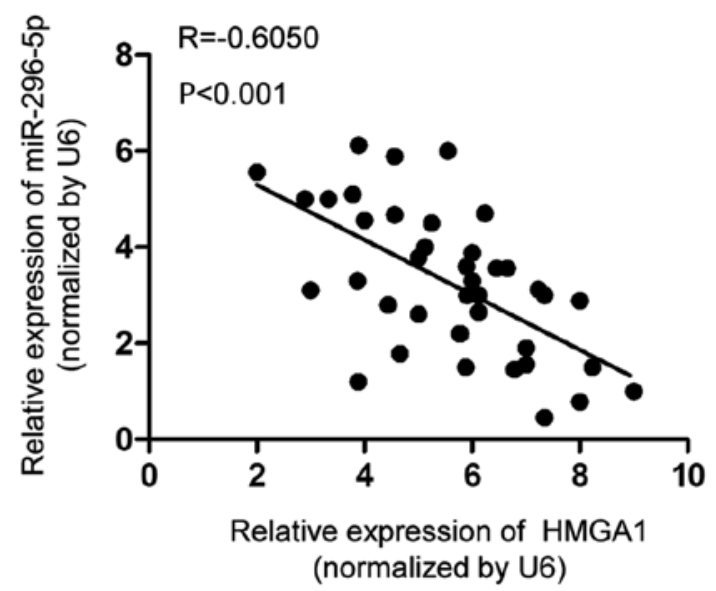

D

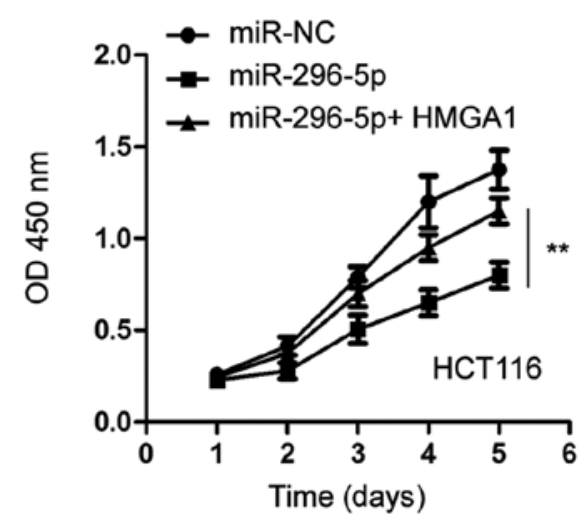

$\mathbf{F}$

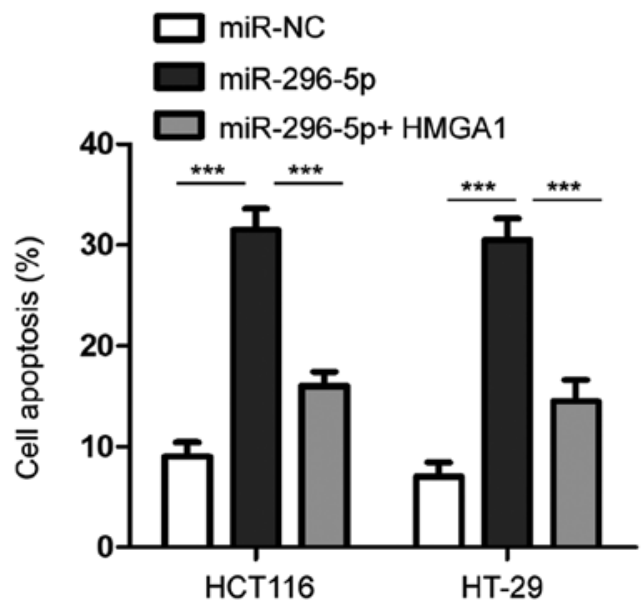

Figure 4. Restoration of HMGA1 reverses the inhibitory effect of miR-296-5p on the growth of CRC cells. (A) The expression level of HMGA1 in paired $\mathrm{CRC}$ tissues and adjacent normal tissues was detected by reverse transcription-quantitative PCR. ${ }^{* * *} \mathrm{P}<0.001$. (B) The correlation between the expression of miR-296-5p and HMGA1 in CRC tissues was analyzed with the Spearman test. (C) Validation of HMGA1 expression in HCT116 and HT-29 cells transfected with Flag-HMGA1. Overexpression of Flag-HMGA1 significantly reversed the inhibitory role of miR-296-5p in regulating the proliferation of (D) HCT116 and (E) HT-29 cells. ${ }^{* *} \mathrm{P}<0.01$. (F) Transfection with Flag-HMGA1 decreased the cell apoptosis induced by miR-296-5p. ${ }^{* * *} \mathrm{P}<0.001$. HMGA1, high mobility group AT-hook 1; miR/miRNA, microRNA; CRC, colorectal cancer; NC, negative control.

of several miRNAs, including miR-26a, miR-195, miR-625 and miR-142-3p (36-39). miR-26a targets and decreases the expression of HMGA1 and inhibits the migration of osteosarcoma cells (40). HMGA1 has also been identified as a target of miR-214, which suppresses the proliferation, migration and invasion of cervical and colorectal cancer cells (41). Additionally, decreased expression of miR-195 promotes the progression of prostate cancer cells by targeting HMGA1 (37). In the present study, HMGA1 was predicted as one of the targets of miR-296-5p. miR-296-5p bound the 3'-UTR of HMGA1 and inhibited the expression of HMGA1 in CRC cells. A higher abundance of HMGA1 was observed in CRC tissues, which was inversely correlated with the expression of miR-296-5p. The restoration of HMGA1 reversed the inhibitory effect of miR-296-5p on the proliferation of CRC cells. These results suggest the important role of miR-296-5p/HMGA1 axis in regulating the progression of CRC. 
Notably, a recent study showed that miR-296-5p inhibits glioblastoma cell stemness by targeting HMGA1 and Sox 2 (42). The overexpression of Sox 2 has been found in a variety of cancer types and is associated with increased cancer aggressiveness, resistance to chemoradiation therapy and a decreased survival rate (43). Since HMGA1 was identified to be a target of miR-296-5p, it would be interesting to further detect the effect of miR-296-5p on the expression of Sox 2 in CRC. Additionally, HMGA1 has been reported to bind the AT-rich regions of DNA and to regulate the expression of cyclin $\mathrm{D} / \mathrm{E}$, which modulates cell cycle progression (44). A recent study demonstrated that HMGA1 activates the STAT3/cyclo-oxygenase 2 pathway and promotes the malignant behaviors of cancer (45). All these findings suggest the possible mechanism of HMGA1 in regulating the survival of $\mathrm{CRC}$ and merit further investigation. Recently, a nano/technological platform was designed to evaluate the expression of HMGA1b in the peripheral blood of patients with cancer (46). Thus, the influence of miR-296-5p on the secretion of HMGA1 in CRC cells should be determined in future studies.

In conclusion, the present study demonstrated the tumor suppressive function of miR-296-5p in CRC, at least in part by negatively modulating the expression of HMGA1. These results indicated the potential clinical significance of miR-296-5p in the diagnosis and prognosis of CRC.

\section{Acknowledgements}

Not applicable.

\section{Funding}

The present study was supported by the Natural Science Foundation of Fujian Province (grant no. 2015J01531), Overseas Students in Science and Technology Activities, Ministry of Human Resources and Social Security of the People's Republic of China (grant no. 2015), and Fujian Health and Family Planning Commission for Young and Middle-aged Talents Training Project (grant no. 2016-ZQN-87). Joint Health Fund Project of Fujian Science and Technology Department (grant no. 2018J01396) and Youth Foundation Project of Fujian Provincial Health Department (grant no. 2014-2-69).

\section{Availability of data and materials}

The datasets used and/or analyzed during the present study are available from the corresponding author on reasonable request.

\section{Authors' contributions}

GY, SY and YZ designed the study. GY and SY performed most of the experiments and analyzed the data. SL collected the clinical samples and cell lines. WT constructed the luciferase reporter vector. XY performed the bioinformatics analysis. JJW analyzed the data, finished the revision and proof of the manuscript and provided funding support. YZ wrote the manuscript. All authors read and approved the final version of the manuscript.

\section{Ethics approval and consent to participate}

The study was approved by the Ethics Committee of the Zhongshan Hospital, Xiamen University (approval no. 2015032013; Xiamen, China). Consent for participation was obtained from all the patients.

\section{Patient consent for publication}

Not applicable.

\section{Competing interests}

These authors declare that they have no competing interests.

\section{References}

1. Sabit H,Cevik E and Tombuloglu H: Colorectal cancer: The epigenetic role of microbiome. World J Clin Cases 7: 3683-3697, 2019.

2. Fakih MG: Metastatic colorectal cancer: Current state and future directions. J Clin Oncol 33: 1809-1824, 2015.

3. Simon K: Colorectal cancer development and advances in screening. Clin Interv Aging 11: 967-976, 2016.

4. Ambros V: The functions of animal microRNAs. Nature 431: 350-355, 2004.

5. Bartel DP: MicroRNAs: Genomics, biogenesis, mechanism, and function. Cell 116: 281-297, 2004.

6. Mohr AM and Mott JL: Overview of microRNA biology. Semin Liver Dis 35: 3-11, 2015.

7. Fabian MR, Sonenberg N and Filipowicz W: Regulation of mRNA translation and stability by microRNAs. Annu Rev Biochem 79: 351-379, 2010.

8. Valenti MT, Dalle Carbonare L and Mottes M: Role of microRNAs in progenitor cell commitment and osteogenic differentiation in health and disease (Review). Int J Mol Med 41: 2441-2449, 2018.

9. Gentilin E, Degli Uberti E and Zatelli MC: Strategies to use microRNAs as therapeutic targets. Best Pract Res Clin Endocrinol Metab 30: 629-639, 2016.

10. Momtazi AA, Shahabipour F, Khatibi S, Johnston TP, Pirro M and Sahebkar A: Curcumin as a microRNA regulator in cancer: A review. Rev Physiol Biochem Pharmacol 171: 1-38, 2016.

11. Iorio MV and Croce CM: MicroRNA dysregulation in cancer: Diagnostics, monitoring and therapeutics. A comprehensive review. EMBO Mol Med 9: 852, 2017.

12. Asadzadeh Z, Mansoori B, Mohammadi A, Aghajani M, Haji-Asgarzadeh K, Safarzadeh E, Mokhtarzadeh A, Duijf PHG and Baradaran B: microRNAs in cancer stem cells: Biology, pathways, and therapeutic opportunities. J Cell Physiol 234: 10002-10017, 2019

13. Kwak PB, Iwasaki S and Tomari Y: The microRNA pathway and cancer. Cancer Sci 101: 2309-2315, 2010.

14. Farazi TA, Spitzer JI, Morozov P and Tuschl T: miRNAs in human cancer. J Pathol 223: 102-115, 2011.

15. Qu H, Xu W, Huang Y and Yang S: Circulating miRNAs: Promising biomarkers of human cancer. Asian Pac J Cancer Prev 12: 1117-1125, 2011.

16. Hosseinahli N, Aghapour M, Duijf PHG and Baradaran B: Treating cancer with microRNA replacement therapy: A literature review. J Cell Physiol 233: 5574-5588, 2018.

17. Wang B, Lu FY, Shi RH, Feng YD, Zhao XD, Lu ZP, Xiao L, Zhou GQ, Qiu JM and Cheng CE: MiR-26b regulates 5-FU-resistance in human colorectal cancer via down-regulation of Pgp. Am J Cancer Res 8: 2518-2527, 2018.

18. Wang W, He Y, Rui J and Xu MQ: miR-410 acts as an oncogene in colorectal cancer cells by targeting dickkopf-related protein 1 via the Wnt//3-catenin signaling pathway. Oncol Lett 17: 807-814, 2019.

19. Muhammad S, Tang Q, Wei L, Zhang Q, Wang G, Muhammad BU, Kaur K, Kamchedalova T, Gang Z, Jiang Z, et al: miRNA-30d serves a critical function in colorectal cancer initiation, progression and invasion via directly targeting the GNA13 gene. Exp Ther Med 17: 260-272, 2019.

20. Xu C, Li S, Chen T, Hu H, Ding C, Xu Z, Chen J, Liu Z, Lei Z, Zhang HT, et al: miR-296-5p suppresses cell viability by directly targeting PLK1 in non-small cell lung cancer. Oncol Rep 35: 497-503, 2016. 
21. Lee KH, Lin FC, Hsu TI, Lin JT, Guo JH, Tsai CH, Lee YC, Lee YC,Chen CL,Hsiao M, et al: MicroRNA-296-5p (miR-296-5p) functions as a tumor suppressor in prostate cancer by directly targeting Pin1. Biochim Biophys Acta 1843: 2055-2066, 2014.

22. Benson AB III, Venook AP, Al-Hawary MM, Cederquist L, Chen YJ, Ciombor KK, Cohen S, Cooper HS, Deming D, Engstrom PF, et al: NCCN Guidelines Insights: Colon Cancer, Version 2.2018. J Natl Compr Canc Netw 16: 359-369, 2018.

23. Livak KJ and Schmittgen TD: Analysis of relative gene expression data using real-time quantitative PCR and the 2(-Delta Delta C(T)) Method. Methods 25: 402-408, 2001.

24. Wong N and Wang X: miRDB: An online resource for microRNA target prediction and functional annotations. Nucleic Acids Res 43 (D1): D146-D152, 2015.

25. Deng Z, Yan F, Jin Q, Wu J, Liu X and Zheng H: Reversal of multidrug resistance phenotype in human breast cancer cells using doxorubicin-liposome-microbubble complexes assisted by ultrasound. J Control Release 174: 109-116, 2014.

26. Shi DM, Li LX, Bian XY, Shi XJ, Lu LL, Zhou HX, Pan TJ, Zhou J, Fan J and Wu WZ: miR-296-5p suppresses EMT of hepatocellular carcinoma via attenuating NRG1/ERBB2/ERBB3 signaling. J Exp Clin Cancer Res 37: 294, 2018.

27. Maia D, de Carvalho AC, Horst MA, Carvalho AL, Scapulatempo-Neto C and Vettore AL: Expression of miR-296-5p as predictive marker for radiotherapy resistance in early-stage laryngeal carcinoma. J Transl Med 13: 262, 2015.

28. Li T, Lu YY, Zhao XD, Guo HQ, Liu CH, Li H, Zhou L, Han YN, Wu KC, Nie YZ, et al: MicroRNA-296-5p increases proliferation in gastric cancer through repression of Caudal-related homeobox 1. Oncogene 33: 783-793, 2014.

29. LeeH,ShinCH,KimHR,ChoiKHandKimHH:MicroRNA-296-5p promotes invasiveness through downregulation of nerve growth factor receptor and Caspase-8. Mol Cells 40: 254-261, 2017.

30. Conte A,Paladino S, Bianco G, Fasano D, Gerlini R, Tornincasa M, Renna M, Fusco A, Tramontano D and Pierantoni GM: High mobility group A1 protein modulates autophagy in cancer cells. Cell Death Differ 24: 1948-1962, 2017.

31. Liu L, Zhang S, Hu L, Liu L, Guo W and Zhang J: HMGA1 participates in MHCC $97 \mathrm{H}$ cell proliferation and invasion through the ILK/Akt/GSK3 $\beta$ signaling pathway. Mol Med Rep 16: 9287-9294, 2017.

32. Colamaio M, Tosti N, Puca F, Mari A, Gattordo R, Kuzay Y, Federico A, Pepe A, Sarnataro D, Ragozzino E, et al: HMGA1 silencing reduces stemness and temozolomide resistance in glioblastoma stem cells. Expert Opin Ther Targets 20: 1169-1179, 2016.

33. Kim DK, Seo EJ, Choi EJ, Lee SI, Kwon YW, Jang IH, Kim SC, Kim KH, Suh DS, Seong-Jang K, et al: Crucial role of HMGA1 in the self-renewal and drug resistance of ovarian cancer stem cells. Exp Mol Med 48: e255, 2016.
34. Quintavalle C, Burmeister K, Piscuoglio S, Quagliata L, Karamitopoulou E, Sepe R, Fusco A, Terracciano LM, Andersen JB, Pallante $\mathrm{P}$, et al: High mobility group A1 enhances tumorigenicity of human cholangiocarcinoma and confers resistance to therapy. Mol Carcinog 56: 2146-2157, 2017.

35. Loria R, Laquintana V, Bon G, Trisciuoglio D, Frapolli R, Covello R, Amoreo CA, Ferraresi V, Zoccali C, Novello M, et al: HMGA1/E2F1 axis and NFkB pathways regulate LPS progression and trabectedin resistance. Oncogene 37: 5926-5938, 2018.

36. Lin Y, Chen H, Hu Z, Mao Y, Xu X, Zhu Y, Xu X, Wu J, Li S, Mao Q, et al: miR-26a inhibits proliferation and motility in bladder cancer by targeting HMGA1. FEBS Lett 587: 2467-2473, 2013.

37. Zhang X, Tao T, Liu C, Guan H, Huang Y, Xu B and Chen M: Downregulation of miR-195 promotes prostate cancer progression by targeting HMGA1. Oncol Rep 36: 376-382, 2016

38. Zhou WB, Zhong CN, Luo XP, Zhang YY, Zhang GY, Zhou DX and Liu LP: miR-625 suppresses cell proliferation and migration by targeting HMGA1 in breast cancer. Biochem Biophys Res Commun 470: 838-844, 2016.

39. Jia XP, Meng LL, Fang JC, Wang HW, Chen J, Zhou J, Wang $\mathrm{CN}$ and Jiang WF: Aberrant expression of miR-142-3p and its target gene HMGA1 and FZD7 in breast cancer and its clinical significance. Clin Lab 64: 915-921, 2018.

40. Liu J, Mi B, Wang Y, Shi C, Mi X, Lu Y and Yu P: miR-26a suppresses osteosarcoma migration and invasion by directly targeting HMGA1. Oncol Lett 15: 8303-8310, 2018.

41. Chandrasekaran KS, Sathyanarayanan A and Karunagaran D: MicroRNA-214 suppresses growth, migration and invasion through a novel target, high mobility group AT-hook 1, in human cervical and colorectal cancer cells. Br J Cancer 115: 741-751, 2016.

42. Lopez-Bertoni H, Lal B, Michelson N, Guerrero-Cázares H, Quiñones-Hinojosa A, Li Y and Laterra J: Epigenetic modulation of a miR-296-5p:HMGA1 axis regulates Sox 2 expression and glioblastoma stem cells. Oncogene 35: 4903-4913, 2016.

43. Chaudhary S, Islam Z, Mishra V, Rawat S, Ashraf GM and Kolatkar PR: Sox2: A Regulatory Factor in Tumorigenesis and Metastasis. Curr Protein Pept Sci 20: 495-504, 2019.

44. Xi Y, Li YS and Tang HB: High mobility group A1 protein acts as a new target of Notch1 signaling and regulates cell proliferation in T leukemia cells. Mol Cell Biochem 374: 173-180, 2013.

45. Belton A, Xian L, Huso T, Koo M, Luo LZ, Turkson J, Page BD, Gunning PT, Liu G, Huso DL, et al: STAT3 inhibitor has potent antitumor activity in B-lineage acute lymphoblastic leukemia cells overexpressing the high mobility group A1 (HMGA1)-STAT3 pathway. Leuk Lymphoma 57: 2681-2684, 2016.

46. Capo A, Sepe R, Pellino G, Milone M, Malapelle U, Pellecchia S, Pepe F, Cacciola NA, Manigrasso M, Bruzzaniti S, et al: Setting up and exploitation of a nano/technological platform for the evaluation of HMGA1b protein in peripheral blood of cancer patients. Nanomedicine (Lond) 15: 231-242, 2019. 